\title{
Dual-polarization NFDM transmission using distributed Raman amplification and NFT- domain equalization
}

\author{
Gaiarin, Simone; Da Ros, Francesco; De Renzis, Nicola; da Silva, Edson P.; Zibar, Darko
}

Published in:

IEEE Photonics Technology Letters

Link to article, DOI:

10.1109/LPT.2018.2874204

Publication date:

2018

Document Version

Peer reviewed version

Link back to DTU Orbit

Citation (APA):

Gaiarin, S., Da Ros, F., De Renzis, N., da Silva, E. P., \& Zibar, D. (2018). Dual-polarization NFDM transmission using distributed Raman amplification and NFT-domain equalization. IEEE Photonics Technology Letters, 30(22), 1983-1986. https://doi.org/10.1109/LPT.2018.2874204

\section{General rights}

Copyright and moral rights for the publications made accessible in the public portal are retained by the authors and/or other copyright owners and it is a condition of accessing publications that users recognise and abide by the legal requirements associated with these rights.

- Users may download and print one copy of any publication from the public portal for the purpose of private study or research.

- You may not further distribute the material or use it for any profit-making activity or commercial gain

- You may freely distribute the URL identifying the publication in the public portal 


\title{
Dual-polarization NFDM transmission using distributed Raman amplification and NFT-domain equalization
}

\author{
Simone Gaiarin, Member, IEEE, OSA, Francesco Da Ros, Member, IEEE, OSA, Nicola De Renzis, \\ Edson P. da Silva, Member, IEEE, and Darko Zibar, Member, IEEE
}

\begin{abstract}
Transmission systems based on the nonlinear Fourier transform (NFT) can potentially address the limitations in transmission reach and throughput set forth by the onset of Kerr-induced nonlinear distortion. Whereas this technique is at a preliminary research stage, a rapid progress has been shown over the past few years leading to experimental demonstrations of dual-polarization systems carrying advanced modulation formats. The lossless transmission required by the NFT to ensure the theoretical validity of the scheme is a fairly strong requirement considering practical transmission links. Here, we address it by using optimized distributed Raman amplification to minimize the power variations to approx. $3 \mathrm{~dB}$ over $200 \mathrm{~km}$, thus approaching the lossless transmission requirement. Additionally we experimentally evaluate the improvement provided by equalization schemes applied to the signals in the nonlinear Fourier domain. By combining distributed Raman amplification and nonlinear-Fourier-domain equalization we show transmission reaches for dual-polarization nonlinear frequency division multiplexing (NFDM) systems transmitting both two eigenvalues (8 bit/symbol) up to $2200 \mathrm{~km}$ and three eigenvalues (12 bit/symbol) up to more than $600 \mathrm{~km}$ at hard-decision (HD) and soft-decision (SD) forward error correction (FEC) threshold, respectively.
\end{abstract}

Index Terms-nonlinear frequency division multiplexing, Raman amplification, equalization, nonlinear Fourier transform

\section{INTRODUCTION}

O PTICAL transmission systems have been steadily improving in transmission rate and spectral efficiency over the past years. The significant progress achieved, however, has made the impact of Kerr nonlinearity in the transmission links one of the dominant impairments, making further improvement extremely challenging. Therefore, a strong research effort has been directed towards digital [1], all-optical [1] and joint optical and digital techniques [1], [2] that enable compensation and mitigation of the nonlinear distortion experienced by the signal during transmission. Whereas promising demonstrations have been shown using these approaches, they all focus on pre- or post-compensation of the nonlinear distortion instead of finding a way to constructively include in the design of the transmission system. Recently, a new technique, which tailors the signaling scheme to the nonlinear channel, has been rapidly attracting attention [3]-[7]. This method makes

S. Gaiarin, F. Da Ros, N. De Renzis, E.P. da Silva, and D. Zibar are with the Department of Photonics Engineering, Technical University of Denmark, Kongens Lyngby, 2800 Denmark, e-mail: \{simga,fdro,nidre,dazi\}@ fotonik.dtu.dk

E.P. da Silva is now with the Department of Electrical Engineering of the Federal University of Campina Grande (UFCG), Paraba, Brazil, email:edson.silva@dee.ufcg.edu.br use of the inverse scattering transform [8], which is more commonly known in the optical communication community as nonlinear Fourier transform (NFT). The theory behind the NFT relies on the fact that the nonlinear Schrödinger equation (NLSE) governing the light propagation through optical fibers belongs to a family of integrable nonlinear partial differential equations (when loss and high-order dispersion are neglected) to which a spectral problem can be associated [8]. By using the NFT and its inverse transformation (INFT), information can be encoded on the spectral components of the signals in the nonlinear-Fourier-domain. The components experience a linear evolution during the transmission through the optical fiber. Furthermore, the fact that two different spectral components (continuous and discrete parts) are associated with a timedomain waveform may potentially provide additional degrees of freedom to design signalling schemes that enable increasing the overall throughput. A number of very impressive demonstrations have already been reported [3]-[5], [7], [9], [10], showing rapid improvements on the modulation techniques, leading to recent demonstrations of a dual-polarization NFTbased transmission using either the continuous [7], [11], [12] or the discrete spectrum [4]. However, a significant challenge is posed by the lossless condition required for the NLSE to be integrable [8]. Whereas NFT systems based on lumped erbium-doped fiber amplified (EDFA) amplification have been shown [3]-[5], [7], [11], maintaining a constant power profile through the propagation is highly desirable. In [4], a simple comparison between the performance achievable with $40-\mathrm{km}$ and $80-\mathrm{km}$ spans clearly showed the benefits of shorter spans, i.e., lower power variations. Distributed Raman amplification (DRA) can provide a flatter power distribution across the transmission, thus maintaining a higher optical signal-to-noise ratio (OSNR). In [10] a thorough numerical analysis of the advantage of DRA for nonlinear frequency division multiplexing (NFDM) is presented but, so far, only preliminarily experimental demonstrations using simple eigenvalue modulation, i.e., on-off keying modulation, have been reported [9], [13]. Additionally, making use of the NFT to encode the information to be transmitted may require to re-evaluate the standard digital signal processing (DSP) chain at the receiver side, as the properties and statistics of signal and noise in the nonlinear Fourier domain may be different. For example, correlation properties between the various nonlinear-Fourierdomain quantities may be exploited to provide equalization. In [9], [14], the correlation between the eigenvalue displacement 


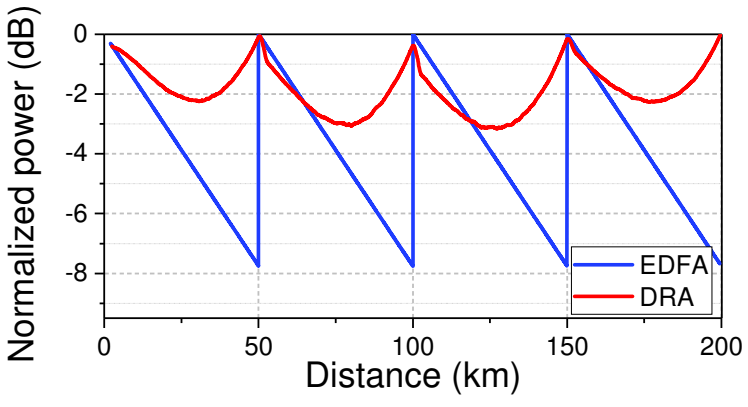

Fig. 1. Power profile across the 200-km transmission link: (red) measured profile with DRA and (blue) simulated profile with EDFA-only amplification.

and the re-scaling and phase-rotation of the spectral amplitudes has been preliminary investigated.

In this letter, we focus on these two aspects, by combining the use of optimized DRA with nonlinear-Fourier-domain equalization for dual-polarization NFDM using quadrature phase shift keying (QPSK) modulation. In particular we experimentally demonstrate the transmission of dual-polarization NFDM signals through a low-loss submarine fiber using DRA with backward pump and evaluate the improvement provided by three different equalization approaches. By minimizing the power variations to approx. $3 \mathrm{~dB}$ over $200-\mathrm{km}$, and applying equalization based on the received eigenvalues, we show a $\mathrm{BER} \leq \mathrm{HD}-\mathrm{FEC}$ threshold after more than 2200-km (2eigenvalue, 8 bit/symbol) and a BER $\leq$ SD-FEC threshold after more than $600 \mathrm{~km}$ (3-eigenvalue, $12 \mathrm{bit} / \mathrm{symbol}$ ).

The letter is organized as follows: in Section III the optimized DRA-based link is described; Section III describes the overall transmission setup with the nonlinear-Fourier-domain equalizer discussed in Section IV the results are discussed in Section $\nabla$ and the conclusions are drawn in Section VI

\section{DRA-AMPLIFIED LINK}

DRA is a well known technique to approach a lossless transmission link. Both forward and backward Raman pumping can be applied. In this work, in order to minimize the noise transfer during amplification, backward-only pumping has been considered [15]. The transmission link is based on four 50-km spans of low-loss and large-effective area SCUBA fiber with an average propagation loss of $0.155 \mathrm{~dB} / \mathrm{km}$, dispersion of $22 \mathrm{ps} / \mathrm{nm} / \mathrm{km}$ and an effective area of $153 \mu \mathrm{m}^{2}$, leading to an estimated nonlinear coefficient $\gamma$ of $0.6 / \mathrm{W} / \mathrm{km}$. DRA with backward pumping is applied at each $50-\mathrm{km}$ span and the pump power has been optimized aiming at minimizing the power variations over each $50-\mathrm{km}$ span, leading to the power profile shown in Fig. 11, measured with an optical time-domain reflectometer. The pumping scheme relied on single-wavelength pumping $(1455 \mathrm{~nm})$ and the power flatness could be further improved by using higher-order pumping [15]. Nevertheless, the scheme chosen enabled to achieve approx. $3 \mathrm{~dB}$ of power variation over the full $200-\mathrm{km}$. These variations are significantly lower than the $8-\mathrm{dB}$ power excursions that would be achieved with standard EDFA-only amplification, even for this low-loss fiber (see Fig. 11).

\section{EXPERIMENTAL SETUP}

The full experimental setup is shown in Fig. 2 At the transmitter side, the pseudo-random bit sequences (PRBSs) are generated and mapped into the NFT coefficients $b\left(\lambda_{i}\right)$, with $\lambda_{i}$ a discrete eigenvalue. For the 2-eigenvalue system, the optimized modulation formats discussed in [4] were used, i.e., $\pi / 4$-shifted QPSK of radius 5 and standard QPSK of radius 0.14 for the $b\left(\lambda_{i}\right)$ associated to the first $\left(\lambda_{1}=0.3 \mathrm{i}\right)$ and second $\left(\lambda_{2}=0.6 \mathrm{i}\right)$ eigenvalue, respectively. The optimization aimed at generating well-confined signals according to the vanishing boundary condition for the NFT, while limiting their peak-to-average power. For the 3-eigenvalue system, all the $b\left(\lambda_{i}\right)\left(\lambda_{i} \in\{0.3 i, 0.6 i, 0.9 i\}\right)$ were modulated using QPSK constellations of radius 1,10 , and 0.01 , respectively. The INFT and following de-normalization provide the waveform in the time domain, with an average power of -2 and $-5 \mathrm{dBm}$, a peak power of 1.7 and $5 \mathrm{~mW}$, and a bandwidth of 3.9 and 5.6 $\mathrm{GHz}$, for the two cases. Note that, due to the limited Raman pump power and in order to satisfy the duration-amplitude constraint of the generated multi-solitons [6], the baud rate was reduced to $250 \mathrm{MBd}$, compared to [4]. The line rate was $2 \mathrm{~Gb} / \mathrm{s}$ and $3 \mathrm{~Gb} / \mathrm{s}$ for the 2- and 3-eigenvalue system, respectively. The digital signals have then been pre-distorted to account for the nonlinear response of the dual-polarization IQ modulator. The four channels of the arbitrary waveform generator (AWG, 64 GSamples/s) drive the modulator, which encodes the dual-polarization NFDM signal into an optical carrier generated by a low-linewidth $(\leq 1 \mathrm{kHz})$ fiber laser, used also as a local oscillator at the receiver side. The signal is transmitted through a recirculating transmission loop based on the link described in Section III Additionally, the loop consists also of an EDFA, which compensates for the loss of the acusto-optical modulators (AOMs) used as optical switches, an optical bandpass filter (OBPF) which suppresses out-ofband amplified spontaneous emission (ASE) noise, and an optical isolator (ISO). After transmission, the signal is sent to a standard pre-amplified coherent receiver based on four balanced photodetectors (BPDs) and a 80-GSamples/s digital storage oscilloscope (DSO) performing the analog-to-digital conversion. The signal polarization is manually aligned at the receiver input for simplicity, alternatively demultiplexing schemes based on training sequences could be implemented as in [11]. The digital waveforms are then processed by offline DSP performing, in order, carrier frequency offset (CFO) compensation to remove the frequency shift introduced by the AOMs, signal amplitude rescaling, low-pass filtering and frame synchronization. A direct NFT is then used to recover the eigenvalues and the NFT coefficients $b\left(\lambda_{i}\right)$. The laser phase noise is compensated by applying blind phase search (BPS) directly on the NFT coefficients $b\left(\lambda_{i}\right)$. This is possible when the expected phase variation is small within a (timedomain) NFDM symbol, and thus the constant phase shift property of the NFT $\exp (i \phi) q(t) \leftrightarrow \exp (-i \phi) b\left(\lambda_{i}\right)$ can be applied [6]. Furthermore, as the eigenvalues have been chosen to be purely imaginary, the phase recovery intrinsically applies the inverse transfer function of the channel, i.e., the exponential phase term $\exp \left(4 i \lambda^{2} L\right)[6]$. After BPS, the 


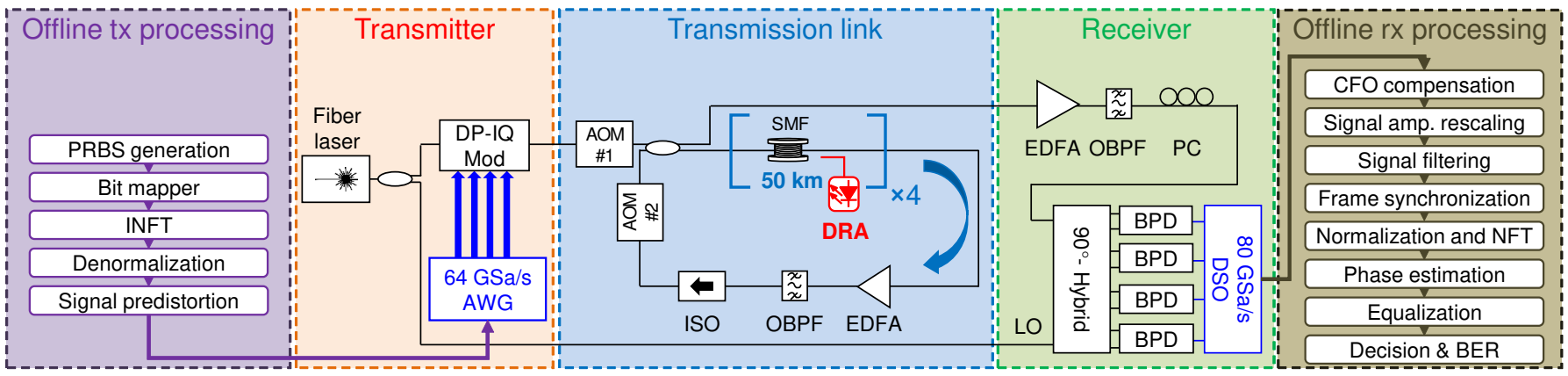

Fig. 2. Experimental setup for the dual-polarization NFDM transmission over a low-loss fiber link with distributed Raman amplification.

nonlinear-Fourier-domain equalization algorithm discussed in Section IV] is applied, decisions are taken on the symbols based on minimum Euclidean distance and bit error counting is performed on more than $10^{6}$ bits, ensuring a reliable bit error ratio (BER) above $10^{-5}$. Throughout this work, the reach is evaluated at the HD-FEC threshold (BER of $3.8 \times 10^{-3}$ ), for the 2-eigenvalue system, and at the SD-FEC threshold (BER of $1.2 \times 10^{-2}$ ), for the 3 -eigenvalue system.

\section{Equalizer}

The time-domain ASE noise and the laser phase noise jointly generate amplitude and phase noise on the NFT coefficients $b\left(\lambda_{i}\right)$ in the NFT domain. To reduce the residual phase noise after BPS, the strong correlation between the noise on the eigenvalues $\lambda_{i}$ and the NFT coefficients $b\left(\lambda_{i}\right)$ can be exploited as suggested in [9] and [14]. In particular, in [9] an equalizer based on linear minimum mean square error (LMMSE) estimation was proposed.

The equalizer aims to estimate the phase and amplitude error terms of the NFT coefficients $b\left(\lambda_{i}\right)$ from selected nonlinearFourier-domain quantities of the observed symbols and compensate for them. In this work, the k-th observed symbol is represented by a feature vector $\mathbf{X}^{(k)}$ that includes a term equal to one and the real and imaginary part of either the eigenvalues $\left(\lambda_{i}\right.$-based equalizer), or the derivative $a^{\prime}\left(\lambda_{i}\right)$ of the NFT coefficient $a\left(\lambda_{i}\right)\left(a^{\prime}\left(\lambda_{i}\right)\right.$-based equalizer), or both of them $\left(\left(\lambda_{i}, a^{\prime}\left(\lambda_{i}\right)\right)\right.$-based equalizer). Each estimator of the $4 \times N$ error terms $\hat{y}_{j}^{(k)}$ (phase and amplitude on each polarization of the $N$ eigenvalues) is characterized by a column vector $\mathbf{n}_{j}$ with as many elements as the considered feature vector $\mathbf{X}^{(k)}$ of an observed symbol. The error $\hat{y}_{j}^{(k)}$ is predicted from the feature vector $\mathbf{X}^{(k)}$ by

$$
\hat{y}_{j}^{(k)}=\mathbf{X}^{(k)} \mathbf{n}_{j} .
$$

The estimator parameter vector $\mathbf{n}_{j}$ is found through linear regression, minimizing the mean squared error (MSE) as in

$$
\mathrm{MSE}=\mathrm{E}\left[\left(\mathbf{y}_{j}-\mathbf{X} \mathbf{n}_{j}\right)^{2}\right],
$$

where $\mathbf{y}_{j}$ is a column vector containing either the phase or amplitude error terms, respectively) of the symbols of different time instances and $\mathbf{X}$ is a matrix whose rows are the feature vectors $\mathbf{X}^{(k)}$ of those symbols. If the inverse of $\mathbf{X}^{T} \mathbf{X}$ exists, the estimator parameter vector $\mathbf{n}$ is unique and given by

$$
\mathbf{n}_{j}=\left(\mathbf{X}^{T} \mathbf{X}\right)^{-1} \mathbf{X}^{T} \mathbf{y}_{j}
$$

where ${ }^{T}$ indicates the transpose of the matrix.

Since this analysis focuses on QPSK constellations, the equalizer is operated in decision directed mode, so that the phase error $\Delta \theta^{(k)}$ is computed directly on the received data by removing the modulation as $\Delta \theta^{(k)}=\left(\frac{1}{4} b\left(\lambda_{i}\right)^{4}-\theta_{0}\right)$ where $\theta_{0}$ is the phase of the reference symbol in the first quadrant.

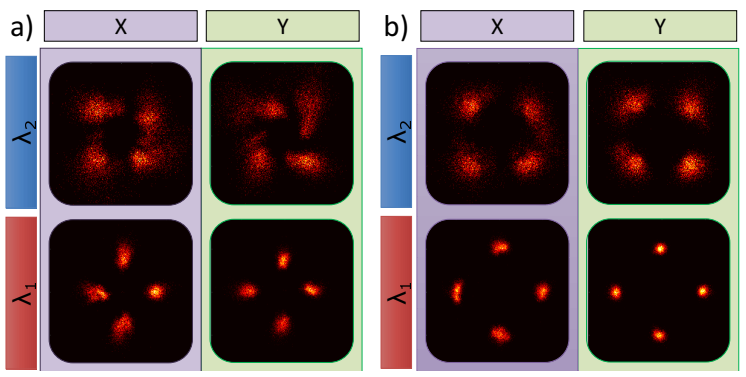

Fig. 3. Constellation diagrams before (a) and after (b) $\lambda_{i}$-based equalization at $2200-\mathrm{km}$ transmission, for the 2-eigenvalue system.

\section{TRANSMISSION RESULTS}

To test the improvement provided by the equalizer, Fig. 3 shows the constellation diagrams before and after $\lambda_{i}$-based equalization for the 2 -eigenvalue system after $2200-\mathrm{km}$ transmission: both phase and amplitude variance are decreased after equalization. The improvement has then been quantified measuring the BER performance as a function of the transmission distance as shown in Fig. 4 for both 2- and 3eigenvalue transmission. In Fig. 4 (a) the total BER for the 2-eigenvalue system is shown and three different LMMSE equalization schemes are benchmarked: (1) using only the real and imaginary part of the eigenvalues $\lambda_{i}$ as features, (2) using only the real and imaginary part of $a^{\prime}\left(\lambda_{i}\right)$ as features, and (3) using both sets $\left(\lambda_{i}, a^{\prime}\left(\lambda_{i}\right)\right)$ as features. As can be seen, any choice of features for the equalization improves the performance at all distances, even though the improvement decreases slightly with the transmission distance. Using directly the information provided by the eigenvalues ( $\lambda_{i}$-based equalizer) provides the best performance out of the three schemes. Fig. 4 (a) shows that going from $\lambda_{i}$-based to $\left(\lambda_{i}, a^{\prime}\left(\lambda_{i}\right)\right)$-based equalization leads to a slight degradation of the performance. This effect may be related to the choice of using a linear equalizer. Since the relation between the quantities involved $b\left(\lambda_{i}\right), a^{\prime}\left(\lambda_{i}\right)$ may not be simply linear, the 

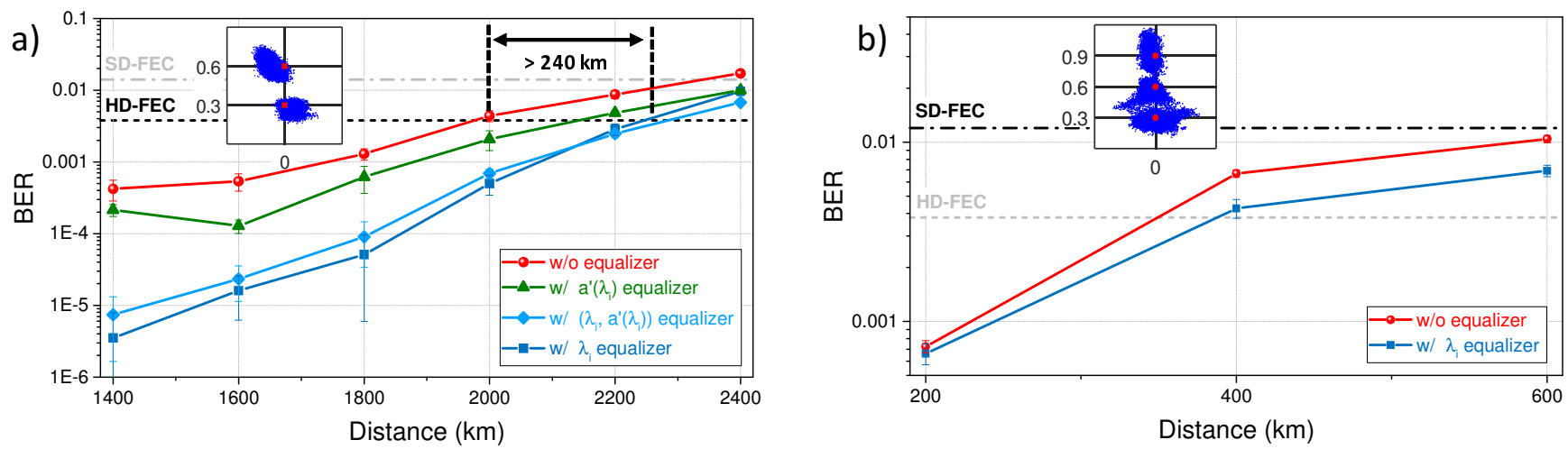

Fig. 4. BER as a function of the transmission distance: (a) comparison between performance without and with $a^{\prime}\left(\lambda_{i}\right),\left(\lambda_{i}, a^{\prime}\left(\lambda_{i}\right)\right)$ and $\lambda_{i}$-based equalization (2eigenvalue system), and (b) comparison without and with $\lambda_{i}$-based equalization (3-eigenvalue system). The insets display the received eigenvalue distributions at $2200 \mathrm{~km}$ for the 2-eigenvalue case and at $600 \mathrm{~km}$ for the 3 -eigenvalue case.

linear fit is believed to perform worse when both $\lambda_{i}$ and $a^{\prime}\left(\lambda_{i}\right)$ are used. By using the $\lambda_{i}$-based equalizer, the improvement provided enables to extend the transmission reach by more than $240 \mathrm{~km}$ at the HD-FEC threshold, resulting in a reach of more than $2200 \mathrm{~km}$. These performances are limited by the worse BER of the higher eigenvalue $\left(\lambda_{2}=0.6 i\right)$, which dominates the overall performance. The BER of the lower eigenvalue $\left(\lambda_{1}=0.3 i\right)$ is instead still well below the HDFEC threshold for all the transmission distances considered, as clearly shown by the constellation diagrams of Fig. 3 . Fig. 4(b), extends the results to the 3-eigenvalue system, again comparing the performance with and without equalization but focusing only on the $\lambda_{i}$-based equalizer. In this case, the transmission distance is significantly reduced, being limited by the worse performance of $\lambda_{3}$, i.e., the eigenvalue with the largest imaginary part [16]. Nevertheless, the equalization still provides a clear improvement and enables a BER well below the SD-FEC threshold for more than $600 \mathrm{~km}$. Note that at high BER values, where the clouds for the different eigenvalues start overlapping (Fig 4(b), inset), errors may be introduced by the wrong classification of the eigenvalues, here performed with a simple k-means algorithm. When the eigenvalues are wrongly classified, the improvement provided by the equalization is reduced by the inaccurate estimation of the cross-correlation. Finally, it was observed that both amplitude and phase errors on $b\left(\lambda_{i}\right)$ are correlated with the imaginary part of $\lambda_{i}$, similarly with the results in [9], [14]. Further investigations of the equalizer performance are ongoing.

\section{CONCLUSION}

We have investigated the transmission performance of a dual-polarization NFDM system using distributed Raman amplification and nonlinear-Fourier-domain equalization. By using low-loss transmission fiber and DRA with backward pumping, the power variations of a full $4 \times 50-\mathrm{km}$ transmission link are kept below $3.1 \mathrm{~dB}$. Furthermore, different nonlinearFourier-domain equalization schemes are evaluated, highlighting that a significant improvement can be obtained by exploiting only the correlation between noise on the eigenvalues and that on the NFT coefficients $b\left(\lambda_{i}\right)$. This enables a BER below the HD-FEC threshold for transmission distance up to $2200 \mathrm{~km}$ for a 2-eigenvalue system and below the SD-FEC threshold at more than to $600 \mathrm{~km}$ for the 3-eigenvalue system.

\section{ACKNOWLEDGMENT}

This work is supported by the European Research Council (ERC-CoG FRECOM project, 771878). We thank OFS fitel Denmark for providing the SCUBA fiber used in this work.

\section{REFERENCES}

[1] A.D. Ellis, et al., "Performance limits in optical communications due to fiber nonlinearity," Adv. Opt. Photonics 9(3), 429-503 (2017).

[2] M.P. Yankov, et al., "Experimental comparison of probabilistic shaping with online PMF optimization and mid-link OPC," in Proc. CLEO 2018 , paper STu4C.2.

[3] S.T. Le, et al., "Nonlinear signal multiplexing for communication beyond the Kerr nonlinearity limit" Nat. Phot., 11, 570-576 (2017).

[4] S. Gaiarin, et al., "Dual-polarization nonlinear Fourier transform-based optical communication system" Optica 5(3), 263-270 (2017).

[5] J.E. Prilepsky, et al., "Nonlinear inverse synthesis and eigenvalue division multiplexing in optical fiber channels," Phys. Rev. Lett. 113, 013901 (2014).

[6] M.I. Yousefi and F.R. Kschischang, "Information transmission using the nonlinear Fourier transform, part I: mathematical tools," IEEE Trans. Inf. Theory 60, 4312-4328 (2014).

[7] J.-W. Goossens, et al., "Polarization-division multiplexing based on the nonlinear Fourier transform," Opt. Express 25(22), 26437-26452 (2017).

[8] V.E. Zakharov and A.B. Shabat, "Exact theory of 2-dimensional selffocusing and one-dimensional self-modulation of waves in nonlinear media," Sov. Phys. JETP 34, 62-69 (1972).

[9] T. Gui, et al., "Alternative decoding methods for optical communications based on nonlinear Fourier transform," J. Light. Technol. 35(9), 15421550 (2017).

[10] S.T. Le, et al., "Nonlinear inverse synthesis for optical links with distributed Raman amplification,” J. Light. Technol. 34(8), 1778-1786 (2016).

[11] T. Gui, et al., "Polarization-division-multiplexed nonlinear frequency division multiplexing," in Proc. CLEO 2018, paper STu4C.3.

[12] S. Civelli, et al., "Polarization-multiplexed nonlinear inverse synthesis with standard and reduced-complexity NFT processing," Opt. Express 26(13), 17360-17377 (2018).

[13] Z. Dong, et al., "Nonlinear frequency division multiplexed transmission based on NFT," Phot. Techn. Lett. 27(15), 1621-1623 (2015).

[14] H. Buelow, et al., "Experimental Nonlinear Frequency Domain Equalization of QPSK Modulated 2-Eigenvalue Soliton," in Proc. OFC 2016, paper Tu2A.3.

[15] M. Tan, et al., "Evaluation of 100G DP-QPSK long-haul transmission performance using second order co-pumped Raman laser based amplification," Opt. Express 23(17), 22181-22189 (2015).

[16] Q. Zhang, et al., "Noise models in the nonlinear spectral domain for optical fibre communications," arXiv:1702.06226 (2017) 